\title{
A New Shuffle Convolution for Multiple Zeta Values
}

AE JA YEE*

yee@math.psu.edu

The Pennsylvania State University, Department of Mathematics, University Park, PA 16802

Received April 17, 2002; Revised January 23, 2004; Accepted January 23, 2004

Abstract. Recently, interest in shuffle algebra has been renewed due to their connections with multiple zeta values. In this paper, we prove a new shuffle convolution that implies a reduction formula for the multiple zeta value $\zeta\left(\{5,1\}^{n}\right)$.

Keywords: multiple zeta values, Euler sums, shuffle algebra, multisets

\section{Introduction}

As in $[4,5,7]$, let $X$ be a finite set and let $X^{*}$ denote the free monoid generated by $X$. We can regard $X$ as an alphabet, and the elements of $X^{*}$ as words formed by concatenating any finite number of letters (repetitions permitted) from $X$.

With the empty word denoted by 1 , it is now possible to define the shuffle product by the recursion

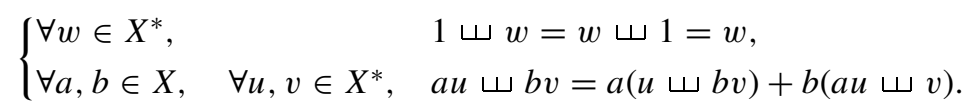

We can easily see that the shuffle product is associative and commutative.

On the other hand, the shuffle product can be defined in terms of permutations. For nonnegative integers $m$ and $n$, let $\operatorname{Shuff}(m, n)$ denote the set of all $\left(\begin{array}{c}m+n \\ n\end{array}\right)$ permutations $\sigma$ of the index set $\{1,2, \ldots, m+n\}$ that satisfy $\sigma^{-1}(j)<\sigma^{-1}(k)$ for all $1 \leq j<k \leq m$ and $m+1 \leq j<k \leq m+n$. Then, the shuffle recursion (1) is easily seen to be equivalent to

$$
\left(\prod_{j=1}^{m} x_{j}\right) \sqcup\left(\prod_{j=m+1}^{m+n} x_{j}\right):=\sum_{\sigma \in \operatorname{Shuff}(m, n)} \prod_{j=1}^{m+n} x_{\sigma(j)}, \quad x_{j} \in X .
$$

The sum (2) is over all non-commutative products (counting multiplicity) of length $m+n$ in which the relative orders of the factors in the products $x_{1} \cdots x_{m}$ and $x_{n+1} \cdots x_{m+n}$ are preserved. The term "shuffle" is used because such permutations arise in riffle shuffling a deck of $m+n$ cards cut into one pile of $m$ cards and a second pile of $n$ cards. Throughout this paper, we will adopt this definition of the shuffles. 
Interest in shuffles has been renewed due to their intimate connections with multiple zeta values [1, 2, 5-10, 12] and multiple polylogarithms [3, 4, 13]. For example, in [2] it was shown that the shuffle convolution formula

$$
\sum_{r=-n}^{n}(-1)^{r}\left[(a b)^{n-r} \uplus(a b)^{n+r}\right]=\left(4 a^{2} b^{2}\right)^{n}, \quad 0 \leq n \in \mathbb{Z},
$$

implies the evaluation

$$
\zeta\left(\{3,1\}^{n}\right):=\zeta(\underbrace{3,1, \ldots, 3,1}_{2 n \text { arguments }})=\frac{2 \pi^{4 n}}{(4 n+2) !}, \quad 0 \leq n \in \mathbb{Z},
$$

for the multiple zeta function defined by

$$
\zeta\left(s_{1}, \ldots, s_{k}\right):=\sum_{n_{1}>\cdots>n_{k}>0} \prod_{j=1}^{k} n_{j}^{-s_{j}}
$$

The primary purpose of this paper is to prove a conjecture of D. Bowman on shuffles (Conjecture 1 below). It follows from our proof of Conjecture 1 that (communication from Bowman)

$$
\zeta\left(\{5,1\}^{n}\right):=\zeta(\underbrace{5,1, \ldots, 5,1}_{2 n \text { arguments }}), \quad 0 \leq n \in \mathbb{Z},
$$

can be explicitly evaluated. Unfortunately, this evaluation has not been communicated to us.

Let $n$ be a positive integer. We begin by observing that in $\left(a^{4} b^{2}\right)^{n}$, every occurrence of $a^{4}$ after the first is separated on both sides by $b^{2}$, and hence there are $2 n-1$ ways in which a single transposition of a letter $b$ with an adjacent letter $a$ can be performed. If we let

$$
\Xi_{n}(k)
$$

denote the sum of the $\left(\begin{array}{c}2 n-1 \\ k\end{array}\right)$ words obtained from $\left(a^{4} b^{2}\right)^{n}$ by making $k$ such transpositions, then we have the following result.

Conjecture 1 (D. Bowman) Let $n$ be a positive integer. Then

$$
\sum_{r=-n}^{n}(-1)^{r}\left[\left(a^{2} b\right)^{n-r} \uplus\left(a^{2} b\right)^{n+r}\right]=3^{n} \sum_{k=0}^{2 n-1} 2^{2 n-k} \Xi_{n}(k) .
$$


Example. When $n=2$, the right hand side of Theorem 1 is equal to

$$
\begin{gathered}
3^{2} \sum_{k=0}^{3} 2^{4-k} \Xi_{2}(k)=144 a^{4} b^{2} a^{4} b^{2}+72\left(a^{4} b^{2} a^{3} b a b+a^{4} b a b a^{3} b^{2}+a^{3} b a b a^{4} b^{2}\right) \\
+36\left(a^{3} b a b a^{3} b a b+a^{3} b a^{2} b a^{3} b^{2}+a^{4} b a b a^{2} b a b\right)+18 a^{3} b a^{2} b a^{2} b a b,
\end{gathered}
$$

which implies that

$$
\begin{aligned}
144 \zeta\left(\{5,1\}^{2}\right)= & \sum_{r=-2}^{2}(-1)^{r} \zeta\left(\{3\}^{2-r}\right) \zeta\left(\{3\}^{2+r}\right)-72(\zeta(5,1,4,2)+\zeta(5,2,4,1) \\
& +\zeta(4,2,5,1))-36(\zeta(4,2,4,2)+\zeta(4,3,4,1)+\zeta(5,2,3,2)) \\
& -18 \zeta(4,3,3,2)
\end{aligned}
$$

The identity (4) is a special case of (12) below.

In Section 2, we present notation and establish some propositions which we use throughout this paper. Conjecture 1 is proved and restated in terms of multiple zeta values in Section 3.

\section{Preliminaries}

Before proving the shuffle convolution formula (3), we need to establish some additional notation and results.

Henceforth, we adopt the convention that concatenation takes precedence over the shuffle operation, so that in (2) the parentheses can be omitted. Also, it will be convenient to denote the multi-set of words arising in the shuffle product (2) by

$$
\left\{x_{1} \cdots x_{m} \sqcup x_{m+1} \cdots x_{m+n}\right\}
$$

For example, $\{a b \sqcup a\}=\{a b a, a a b, a a b\}$. From (2), it is clear that to every distinct word

$$
w \in\left\{\prod_{j=1}^{m} x_{j} \sqcup \prod_{j=m+1}^{m+n} x_{j}\right\},
$$

there corresponds at least one permutation $\sigma \in \operatorname{Shuff}(m, n)$ such that

$$
w=\prod_{j=1}^{m+n} x_{\sigma(j)} .
$$

We call such a permutation $\sigma$ a shuffle corresponding to $w$.

In [11], D. Loeb introduced hybrid sets, which is defined as follows. 
Definition 1 (D. Loeb [11]) Given a universe $U$, any function $f: U \rightarrow \mathbb{Z}$ is called a hybrid set. The value of $f(x)$ is said to be the multiplicity of the element $x$. If $f(x) \neq 0$, we say $x$ is a member of $f$ and write $x \in f$; otherwise, we write $x \notin f$. Define the number of elements \#f to be the sum $\sum_{x \in U} f(x)$. Then $f$ is said to be an \#f hybrid set.

Hybrid sets are denoted by $\{\mid\}$, where elements occurring with positive multiplicities are written on the right of the bar, and elements occurring with negative multiplicities are written on the left. Order is completely irrelevant. For example, if $f=\{d, e, e \mid a, b, c, b\}$, then $f(a)=1, f(b)=2, f(c)=1, f(d)=-1, f(e)=-2$, and $f(x)=0$ for $x \neq$ $a, b, c, d, e$.

Note that in Loeb's paper, elements occurring with positive multiplicities appear on the left of the bar and elements occurring with negative multiplicities appear on the right of the bar, but we follow the definition above throughout this paper.

Loeb did not discuss the product of hybrid sets. We define the product of hybrid sets as follows.

Definition 2 Consider two hybrid sets $f: U \rightarrow \mathbb{Z}$ and $g: V \rightarrow \mathbb{Z}$. We define the product $U \times V$ of $U$ and $V$ as the set of elements obtained by concatenating $x \in U$ and $y \in V$,i.e.,

$$
U \times V=\{x y: x \in U, y \in V\} .
$$

The product $f \times g$ of $f$ and $g$ is a hybrid set from $U \times V$ to $\mathbb{Z}$ defined by

$$
f \times g(x y)=f(x) g(y),
$$

where $x \in f$ and $y \in g$.

From the definition of the number of elements of a hybrid set, the number of elements $\# f \times g$ is defined by

$$
\#(f \times g)=\sum_{x y \in U \times V} f \times g(x y),
$$

which is equal to

$$
\sum_{x \in U, y \in V} f(x) g(y)=\sum_{x \in U} f(x) \sum_{y \in V} g(y)=(\# f)(\# g) .
$$

Similarly, we can generalize the product of two hybrid sets to more than two hybrid sets.

If $w$ is any word, let $|w|$ denote its length, that is, the number of letters in $w$, counting multiplicity. For $w \in\{a, b\}^{*}$, we denote the number of occurrences of the letter $a$ in $w$ by $|w|_{a}$. Similarly, the number of occurrences of the letter $b$ in $w$ is denoted by $|w|_{b}$, so that $|w|=|w|_{a}+|w|_{b}$. For $1 \leq j \leq|w|_{b}$, let $b_{j}=b_{j}(w)$ be the $j$ th $b$ from the left; let $a(1)$ be the number of times the letter $a$ occurs to the left of $b_{1}$; and for $1<j \leq|w|_{b}$ let $a(j)$ be the number of times the letter $a$ occurs between $b_{j-1}$ and $b_{j}$. Let $A(w)$ denote the sequence $a(1), a(2), \ldots, a\left(|w|_{b}\right)$. 
Proposition 1 Let $X$ be an alphabet, let $m$ and $n$ be non-negative integers, and let

$$
w \in\left\{\prod_{j=1}^{m} x_{j} \uplus \prod_{j=m+1}^{m+n} x_{j}\right\}, \quad x_{j} \in X
$$

If $k$ is an integer such that $0 \leq k \leq m+n$, and $w=u v$ with $|u|=k$, then there exist non-negative integers $m_{1}$ and $n_{1}$ satisfying $m_{1}+n_{1}=k$ and such that

$$
u \in\left\{\prod_{j=1}^{m_{1}} x_{j} \uplus \prod_{j=m_{1}+1}^{m_{1}+n_{1}} x_{m-m_{1}+j}\right\}
$$

and

$$
v \in\left\{\prod_{j=1}^{m-m_{1}} x_{m_{1}+j} \uplus \prod_{j=m-m_{1}+1}^{m-m_{1}+n-n_{1}} x_{m_{1}+n_{1}+j}\right\}
$$

Conversely, given non-negative integers $m_{1}, m_{2}, n_{1}$, and $n_{2}$, let

$$
u \in\left\{\prod_{j=1}^{m_{1}} x_{j} \uplus \prod_{j=m_{1}+1}^{m_{1}+n_{1}} x_{j}\right\} \quad \text { and } \quad v \in\left\{\prod_{j=1}^{m_{2}} y_{j} \amalg \prod_{j=m_{2}+1}^{m_{2}+n_{2}} y_{j}\right\}, \quad x_{j}, y_{j} \in X .
$$

Then

$$
u v \in\left\{\left(\prod_{j=1}^{m_{1}} x_{j}\right)\left(\prod_{j=1}^{m_{2}} y_{j}\right) \uplus\left(\prod_{j=m_{1}+1}^{m_{1}+n_{1}} x_{j}\right)\left(\prod_{j=m_{2}+1}^{m_{2}+n_{2}} y_{j}\right)\right\}
$$

Proof: Let $\sigma$ be a shuffle corresponding to $w$. Then

$$
u=\prod_{j=1}^{k} x_{\sigma(j)} \quad \text { and } \quad v=\prod_{j=k+1}^{m+n} x_{\sigma(j)} .
$$

Let $m_{1}=|\{j \in \mathbb{Z}: 1 \leq j \leq k, \sigma(j) \leq m\}|$, and put $n_{1}=k-m_{1}$. Note that if $1 \leq j \leq k$ and $\sigma(j) \leq m$, then $\sigma(j) \leq m_{1}$, since $\sigma \in$ Shuff $(m, n)$. Similarly, $1 \leq j \leq k$ and $m+1 \leq \sigma(j) \leq m+n$ implies $m+1 \leq \sigma(j) \leq m+n_{1}$. Hence, we can define a permutation $\tau$ on $\{1,2, \ldots, k\}$ by

$$
\tau(j)= \begin{cases}\sigma(j), & \text { if } 1 \leq \sigma(j) \leq m, \\ \sigma(j)-m+m_{1}, & \text { if } m+1 \leq \sigma(j) \leq m+n\end{cases}
$$


Similarly, we can define a permutation $\rho$ on $\{1,2, \ldots, m+n-k\}$ by

$$
\rho(j)= \begin{cases}\sigma(j+k)-m_{1}, & \text { if } 1 \leq \sigma(j+k) \leq m, \\ \sigma(j+k)-k, & \text { if } m+1 \leq \sigma(j+k) \leq m+n\end{cases}
$$

Since $\sigma \in \operatorname{Shuff}(m, n)$, we see that $\tau \in \operatorname{Shuff}\left(m_{1}, n_{1}\right)$ and $\rho \in \operatorname{Shuff}\left(m-m_{1}, n-n_{1}\right)$. Let $z_{j}=x_{j}$ for $1 \leq j \leq m_{1}$ and $z_{j}=x_{m-m_{1}+j}$ for $m_{1}+1 \leq j \leq m_{1}+n_{1}$. Then

$$
u=\prod_{j=1}^{k} z_{\tau(j)}
$$

Since $\tau \in \operatorname{Shuff}\left(m_{1}, n_{1}\right)$, it follows that

$$
u \in\left\{\prod_{j=1}^{m_{1}} z_{j} \sqcup \prod_{j=m_{1}+1}^{m_{1}+n_{1}} z_{j}\right\}
$$

which is equivalent to (5). Similarly, we can show that $\rho$ is a shuffle corresponding to $v$ in the shuffle product (6).

For the converse, let $\tau \in \operatorname{Shuff}\left(m_{1}, n_{1}\right)$ and $\rho \in \operatorname{Shuff}\left(m_{2}, n_{2}\right)$ be shuffles corresponding to $u$ and $v$, respectively. Then

$$
u=\prod_{j=1}^{m_{1}+n_{1}} x_{\tau(j)} \quad \text { and } \quad v=\prod_{j=1}^{m_{2}+n_{2}} y_{\rho(j)}
$$

We define a permutation $\sigma$ on $\left\{1,2, \ldots, m_{1}+n_{1}+m_{2}+n_{2}\right\}$ by

$$
\sigma(j)= \begin{cases}\tau(j), & \text { if } 1 \leq \tau(j) \leq m_{1}, \\ \tau(j)+m_{2}, & \text { if } m_{1}+1 \leq \tau(j) \leq m_{1}+n_{1},\end{cases}
$$

for $1 \leq j \leq m_{1}+n_{1}$, and

$$
\sigma(j)= \begin{cases}\rho\left(j-m_{1}-n_{1}\right)+m_{1}, & \text { if } 1 \leq \rho\left(j-m_{1}-n_{1}\right) \leq m_{2}, \\ \rho\left(j-m_{1}-n_{1}\right)+m_{1}+n_{1}, & \text { if } m_{2}+1 \leq \rho\left(j-m_{1}-n_{1}\right) \leq m_{2}+n_{2},\end{cases}
$$

for $m_{1}+n_{1}+1 \leq j \leq m_{1}+n_{1}+m_{2}+n_{2}$. Since $\tau \in \operatorname{Shuff}\left(m_{1}, n_{1}\right)$ and $\rho \in \operatorname{Shuff}\left(m_{2}, n_{2}\right)$, it follows that $\sigma \in \operatorname{Shuff}\left(m_{1}+m_{2}, n_{1}+n_{2}\right)$. Let

$$
z_{j}= \begin{cases}x_{j}, & \text { if } 1 \leq j \leq m_{1}, \\ y_{j-m_{1}}, & \text { if } m_{1}+1 \leq j \leq m_{1}+m_{2}, \\ x_{j-m_{2}}, & \text { if } m_{1}+m_{2}+1 \leq j \leq m_{1}+m_{2}+n_{1}, \\ y_{j-m_{1}-n_{1}}, & \text { if } m_{1}+m_{2}+n_{1}+1 \leq j \leq m_{1}+m_{2}+n_{1}+n_{2} .\end{cases}
$$


Then

$$
u v=\prod_{j=1}^{m_{1}+n_{1}+m_{2}+n_{2}} z_{\sigma(j)}
$$

Since $\sigma \in \operatorname{Shuff}\left(m_{1}+m_{2}, n_{1}+n_{2}\right)$,

$$
u v \in\left\{\prod_{j=1}^{m_{1}+m_{2}} z_{j} \uplus \prod_{j=m_{1}+m_{2}+1}^{m_{1}+m_{2}+n_{1}+n_{2}} z_{j}\right\},
$$

which is equivalent to (7).

In the remainder of our paper, $X$ has only two elements, $a$ and $b$. We need to make further definitions, to study carefully the formation of words in the shuffle convolution (3), and to carefully examine words in $X^{*}$.

Definition 3 For a non-negative integer $n$, let $\Pi_{n}$ be the set of all non-negative integer sequences $\pi=\left(\pi_{1}, \pi_{2}, \ldots, \pi_{n}\right)$ satisfying

$$
2 m \leq \sum_{j=1}^{m} \pi_{j} \leq 2 m+2 \quad \text { for } \quad m<n, \quad \text { and } \quad \sum_{j=1}^{n} \pi_{j}=2 n
$$

Proposition 2 Let $n$ and $r$ be non-negative integers with $n \geq r$, and let

$$
w \in\left\{\left(a^{2} b\right)^{r} \amalg\left(a^{2} b\right)^{n-r}\right\} .
$$

Then $A(w)$ belongs to $\Pi_{n}$.

Conversely, there exists a non-negative integer $r$ with $r \leq n$ such that $w \in\left\{\left(a^{2} b\right)^{n-r} \amalg\right.$ $\left.\left(a^{2} b\right)^{r}\right\}$ for $w \in\{a, b\}^{*}$, and $A(w)$ belongs to $\Pi_{n}$.

Proof: Let $n \geq r, 1 \leq m \leq n, u:=\left(a^{2} b\right)^{r}$, and $v:=\left(a^{2} b\right)^{n-r}$, and suppose $w \in\{u \amalg v\}$. Since each $b$ in any power of $a^{2} b$ is immediately preceded by two consecutive occurrences of the letter $a$, the shuffle rule (2) implies that the number of times the letter $a$ appears to the left of $b_{m}$ is at least $2 m$. In other words,

$$
2 m \leq \sum_{j=1}^{m} a(j)
$$

It is possible that there are up to two additional occurrences of the letter $a$ preceding $b_{m}$. Let $\tau$ be the initial subword of $w$ consisting of the letters from $w_{1}$ up to and including $b_{m}$. In the formation of $\tau$, let $k$ be the number of occurrences of the letter $b$ that came from $u$; then the remaining $m-k$ came from $v$. If $b_{m}$ came from $u$, then $\tau$ was formed from $\left(a^{2} b\right)^{k} \sqcup\left(a^{2} b\right)^{m-k} s$, where $s$ is either $a, a^{2}$, or the empty word. Similarly, if $b_{m}$ 
came from $v$, then $\tau$ was formed from $\left(a^{2} b\right)^{m} s \amalg\left(a^{2} b\right)^{m-k}$. Since the letters of $s$ could precede $b_{m}$ in $\tau$, it follows that

$$
\sum_{j=1}^{m} a(j) \leq 2 m+2
$$

For the converse, first observe that if $w \in\{a, b\}^{*}$ satisfies $|w|_{a}=2|w|_{b}=2 n$ and the system of inequalities (8) holds for $1 \leq m \leq n$, then $2 \leq a(1) \leq 4$ and $0 \leq a(m) \leq 4$ for $1<m \leq n$. Suppose there are precisely $s$ indices $m_{1}<m_{2}<\cdots<m_{s}$ satisfying $0 \leq a\left(m_{k}\right) \leq 1$ for $1 \leq k \leq s$. We may assume $s>0$, for otherwise $|w|_{a}=2|w|_{b}$ implies $w=\left(a^{2} b\right)^{n}$, and the conclusion is trivial. Since

$$
2 m_{1}<\sum_{j=1}^{m_{1}} a(j) \leq 2 m_{1}+2
$$

there exists $m<m_{1}$ for which $3 \leq p:=a(m) \leq 4-a\left(m_{1}\right)$. Choose the maximal such $m$, and remove $p-2$ of the letters $a$ that occur between $b_{m-1}$ and $b_{m}$ (before $b_{1}$ if $m=1$ ), so that in the modified word, $a(m)=2$. If $p=4$, then two letters $a$ are removed; let us also remove $b_{m_{1}}$ so that $a^{2} b$ has been removed. If $p=3$, then only one letter $a$ is removed, and either $a\left(m_{1}\right)=1$, or there exists $m^{\prime}<m$ for which $a\left(m^{\prime}\right)=3$. Now remove also $b_{m_{1}}$ and the $a$ that immediately precedes $b_{m_{1}}$ if $a\left(m_{1}\right)=1$. Otherwise, remove $b_{m_{1}}$ and the $a$ that immediately precedes $b_{m^{\prime}}$, so that now $a\left(m^{\prime}\right)=2$. Then $a^{2} b$ has been removed from $w$. Repeat the process for $m_{2}, \ldots, m_{s}$ until we get $\left(a^{2} b\right)^{n-r}$ left for some $r \leq s$. Note that $\left(a^{2} b\right)^{r}$ is removed from $w$ and $w \in\left\{\left(a^{2} b\right)^{r} \sqcup\left(a^{2} b\right)^{n-r}\right\}$.

\section{The main result}

Definition 4 For each non-negative integer $n$ and each integer $r$ with $|r| \leq n$, let

$$
S_{n, r}:=\left\{\left(a^{2} b\right)^{n-r} \uplus\left(a^{2} b\right)^{n+r}\right\} \quad \text { and } \quad S_{n}:=\left\{\cup_{r \text { odd }} S_{n, r} \mid \cup_{r \text { even }} S_{n, r}\right\} .
$$

Definition 5 For a non-negative integers $n$, let $\Delta_{n}$ be the subset of $\Pi_{2 n}$ such that

$$
\begin{cases}2 m+1 \leq \sum_{j=1}^{m} \pi_{j} \leq 2 m+2, & \text { if } m \text { is odd, } \\ 2 m \leq \sum_{j=1}^{m} \pi_{j} \leq 2 m+1, & \text { if } m \text { is even and less than } 2 n, \\ \sum_{j=1}^{2 n} \pi_{j}=4 n, & \text { if } m=2 n,\end{cases}
$$

and for a non-negative integer $k$, let $\Delta_{n}(k)$ be the subset of $\Delta_{n}$ such that

$$
e(\pi):=\pi_{2}+\pi_{4}+\cdots+\pi_{2 n}=k
$$


Since (9) implies $0 \leq \pi_{2 m} \leq 2$ for any $m<n$ and $0 \leq \pi_{2 n} \leq 1$, we see that $\Delta_{n}(k)$ is empty if $k \geq 2 n$. In other words, we partition $\Delta_{n}$ into $2 n$ subsets $\Delta_{n}(k)$,

$$
\Delta_{n}=\bigcup_{k=0}^{2 n-1} \Delta_{n}(k)
$$

Example. When $n=2$,

$$
\begin{aligned}
& \Delta_{2}(0)=\{(4,0,4,0)\}, \quad \Delta_{2}(1)=\{(4,0,3,1),(4,1,3,0),(3,1,4,0)\} \\
& \Delta_{2}(2)=\{(4,1,2,1),(3,1,3,1),(3,2,3,0)\}, \quad \Delta_{2}(3)=\{(3,2,2,1)\}
\end{aligned}
$$

Theorem 1 Let $n$ be a positive integer. Then

$$
\sum_{r=-n}^{n}(-1)^{r}\left[\left(a^{2} b\right)^{n-r} \uplus\left(a^{2} b\right)^{n+r}\right]=3^{n} \sum_{k=0}^{2 n-1} 2^{2 n-k} \sum_{A(w) \in \Delta_{n}(k)} w .
$$

In the following lemma, we show that Theorem 1 is equivalent to Conjecture 1 , which is restated in terms of multiple zeta values. Using [4], we conclude that

$$
\begin{aligned}
& \sum_{r=-n}^{n}(-1)^{r} \zeta\left(\{3\}^{n-r}\right) \zeta\left(\{3\}^{n+r}\right) \\
& \quad=3^{n} \sum_{k=0}^{2 n-1} 2^{2 n-k} \sum_{\left(s_{1}, s_{2} \ldots, s_{2 n}\right) \in \Delta_{n}(k)} \zeta\left(s_{1}+1, s_{2}+1, \ldots, s_{2 n}+1\right)
\end{aligned}
$$

Lemma 1 Let $n$ and $k$ be integers with $0 \leq k<2 n$. Then

$$
\Xi_{n}(k)=\sum_{A(w) \in \Delta_{n}(k)} w
$$

Proof: Let $\bar{w}:=\left(a^{4} b^{2}\right)^{n}$ and $A(\bar{w}):=(\bar{a}(1), \bar{a}(2), \ldots, \bar{a}(2 n))$, where $\bar{a}(1)$ is the number of times the letter $a$ occurs to the left of $b_{1}$ in $\bar{w}$, and, for $1<j \leq|\bar{w}|_{b}, \bar{a}(j)$ is the number of times the letter $a$ occurs between $b_{j-1}$ and $b_{j}$, i.e.,

$$
\bar{a}(j)= \begin{cases}4, & \text { if } j \text { is odd } \\ 0, & \text { if } j \text { is even }\end{cases}
$$

Note that $\bar{a}(w)$ satisfies (9).

We first show that for a given sequence $\pi \in \Delta_{n}(k)$, we can produce a word $w$ from $\bar{w}$ using the allowed transpositions of $a$ and $b$ in $\bar{w}$. Let $\pi$ be a sequence in $\Delta_{n}(k)$. We will make transpositions within $\bar{w}$ such that the number of $a$ 's on the left of $b_{1}$ becomes $\pi_{1}$ and the number of $a$ 's between $b_{i-1}$ and $b_{i}$ becomes $\pi_{j}$ for $1<i \leq 2 n$. Since $\pi$ satisfies (9), $3 \leq \pi_{1} \leq 4$ and $4 \leq \pi_{1}+\pi_{2} \leq 5$. There are four cases for $\left(\pi_{1}, \pi_{2}\right):(4,0),(4,1),(3,1)$, and 
$(3,2)$. If $\pi_{1}=4$ and $\pi_{2}=0$, then we do not make any transposition of a single $a$ in the first $a^{4}$ and a single $b$ in the first $b^{2}$. If $\pi_{1}=4$ and $\pi_{2}=1$, then we transpose a single $a$ among the second $a^{4}$ and a single $b$ in the first $b^{2}$. If $\pi_{1}=3$ and $\pi_{2}=1$, then we transpose a single $a$ among the first $a^{4}$ and single $b$ in the first $b^{2}$. If $\pi_{1}=3$ and $\pi_{2}=2$, we transpose a single $a$ in the first $a^{4}$ and a single $b$ in the first $b^{2}$, and then transpose a single $a$ in the second $a^{4}$ and the $b$ which was not transposed in the first $b^{2}$. We make transpositions of single $a$ 's and single $b$ 's proceeding from the left in $\bar{w}$ in this way. Then we see that we can only use the allowed transpositions since $\pi$ satisfies (9). Moreover, the number of transpositions we made is $k$ since $\pi \in \Delta_{n}(k)$. Let $w$ be the word with $A(w)=\pi$. Hence, $w$ arises in $\Xi_{n}(k)$.

We now consider the reverse. In other words, we will show the $A(w)$ is in $\Delta_{n}(k)$ for a given $w$ that arises in $\Xi_{n}(k)$. Since $\bar{a}(2 i-1)+\bar{a}(2 i)=4$ for $1 \leq i \leq n$, the transposition of a single $a$ in the $i$ th $a^{4}$ and a single $b$ in the $i$ th $b^{2}$ does not change $\bar{a}(2 i-1)+\bar{a}(2 i)=4$. If a transposition of a single $a$ in the $(i+1)$ st $a^{4}$ and a single $b$ in the $i$ th $b^{2}$ is made, then $\bar{a}(2 i-1)+\bar{a}(2 i)$ becomes 5. Suppose that $w$ is a word arising in $\Xi_{n}(k)$. Since $w$ is obtained from $\bar{w}$ by making $k$ such transpositions, it can be easily shown that $A(w)$, the sequence of the number of $a$ 's between $b$ 's, satisfies (9). Furthermore, whenever a transposition is made, the number of the $a$ 's between the $(2 i-1)$ st $b$ and the $2 i$ th $b$ increases by 1 . Hence, $A(w) \in \Delta_{n}(k)$.

We now prove Theorem 1. In the alternating sum in this theorem, many words are cancelled because they appear with equal coefficients but opposite signs. We characterize the words cancelled in the alternating sum.

Lemma 2 For a positive integer $n$ and an integer $r,|r| \leq n$, let $w$ be a word in $S_{n, r}$ whose $A(w)$ does not belong to $\Delta_{n}$. Then there is a unique $r^{\prime}$ with different parity from $r$ such that the word $w$ belongs to $S_{n, r^{\prime}}$ as well. Furthermore, the number of occurrences of the word $w$ in $S_{n, r}$ is equal to the number of occurrences in $S_{n, r^{\prime}}$.

Proof: Let $m$ be the smallest positive integer for which (9) is not satisfied.

First, suppose that $m$ is odd. Write $w=u v$, where $u$ is the initial subword of $w$ consisting of the letters from $w_{1}$ up to including $b_{m}$. Since $|u|_{a}=2|u|_{b}=2 m$, we infer from Proposition 1 that there exists $s$ with $s \leq m$ and such that

$$
u \in\left\{\left(a^{2} b\right)^{s} \uplus\left(a^{2} b\right)^{m-s}\right\} \quad \text { and } \quad v \in\left\{\left(a^{2} b\right)^{n-r-s} \uplus\left(a^{2} b\right)^{n+r-m+s}\right\} .
$$

Since the shuffle product is commutative, $u^{\prime}=u \in\left\{\left(a^{2} b\right)^{m-s} \uplus\left(a^{2} b\right)^{s}\right\}$ as well. Let $w^{\prime}:=u^{\prime} v$. Then $w^{\prime} \in S_{n, r-m+2 s}$. Since $m$ is odd, the parity of $(r-m+2 s)$ must be opposite to the parity of $r$.

Now we show that the number of occurrences of the word $w$ in $S_{n, r}$ and $S_{n, r-m+2 s}$ are equal. Let $\sigma \in \operatorname{Shuff}(n-r, n+r)$ be a shuffle corresponding to $w$. Then Proposition 1 implies that there exist shuffles $\tau$ and $\rho$ corresponding to $u$ and $v$, respectively.

We define a permutation $\tau^{\prime}$ induced from $\tau$ by

$$
\tau^{\prime}(j)= \begin{cases}\tau(j)+3 m-3 s, & \text { if } 1 \leq \tau(j) \leq 3 s \\ \tau(j)-3 s, & \text { if } 3 s+1 \leq \tau(j) \leq 3 m\end{cases}
$$


It is easily seen that $\tau^{\prime}$ is a shuffle corresponding to $u^{\prime}$, and that $\tau^{\prime}$ and $\rho$ produce a unique shuffle $\sigma^{\prime}$ corresponding to $w^{\prime}$.

On the other hand, suppose that $m$ is even. Write $w=u v$, where $u$ is the initial subword of $w$ consisting of the letters from $w_{1}$ up to but excluding $b_{m}$. Proposition 1 now implies that there exists $s<m$ such that

$$
u \in\left\{\left(a^{2} b\right)^{s} a^{2} \amalg\left(a^{2} b\right)^{m-1-s} a^{2}\right\} \quad \text { and } \quad v \in\left\{b\left(a^{2} b\right)^{n-r-s-1} \sqcup b\left(a^{2} b\right)^{n+r-m+s}\right\} .
$$

Since the shuffle product is commutative, $u^{\prime}=u \in\left\{\left(a^{2} b\right)^{m-1-s} a^{2} \sqcup\left(a^{2} b\right)^{s} a^{2}\right\}$ as well. Let $w^{\prime}:=u^{\prime} v$. Then $w^{\prime} \in S_{n, r-m+1+2 s}$. Since $m$ is even, the parity of $(r-m+1+2 s)$ must be opposite to the parity of $r$.

As we did in the case when $m$ is odd, we can show the number of occurrences of the word $w$ in $S_{n, r}$ and $S_{n, r-m+1+2 s}$ are equal. We omit the details.

Lemma 2 implies that there remain only the words $w$ with $A(w) \in \Delta_{n}$ in the alternating sum in Theorem 1 .

Proof of Theorem 1: Throughout this proof, we assume that any words arising from $\{u \sqcup v\}, u, v \in\{a, b\}^{*}$, have negative and positive multiplicities if the difference of the numbers of $b$ 's in $u$ and $v$ is congruent to 2 and 0 modulo 4 , respectively.

Let $Q_{n, r}$ be a multi-set consisting of those words in $S_{n, r}$ such that $A(w)$ belongs to $\Delta_{n}$, and let $Q_{n}$ be a hybrid set defined by

$$
\left\{\cup_{r \text { odd }} Q_{n, r} \mid \cup_{r \text { even }} Q_{n, r}\right\} \text {. }
$$

By the definition of $S_{n}$, Lemma 2, and the definition of $Q_{n}$, we can rewrite the alternating sum in Theorem 1 as

$$
\sum_{|r| \leq n}(-1)^{r} \sum_{w \in S_{n, r}} w=\sum_{w \in S_{n}} w=\sum_{w \in Q_{n}} w
$$

Since $\Delta_{n}$ has the partition (11), to complete the proof of Theorem 1, it suffices to show that for each $0 \leq k<2 n$,

$$
\sum_{w \in Q_{n}(k)} w=3^{n} 2^{2 n-k} \sum_{A(w) \in \Delta_{n}(k)} w,
$$

where $Q_{n}(k)$ is a hybrid subset consisting of those words of $Q_{n}$ such that $A(w)$ belongs to $\Delta_{n}(k)$.

To this end, let $w$ be a word in $Q_{n}(k)$. Suppose that there are exactly $h=h(w)$ positive integers $m$ that satisfy $\sum_{j=1}^{m} a(j)=2 m$, say, $m_{1}<m_{2}<\cdots<m_{h}=2 n$. Since $w$ satisfies (9), $m_{p}$ must be even for each $p=1,2, \ldots, h$. Let

$$
u_{1}:=\prod_{j=1}^{3 m_{1}} w_{j}, \quad u_{p}:=\prod_{j=3 m_{p-1}+1}^{3 m_{p}} w_{j}, \quad 2 \leq p \leq h
$$


We call $w=u_{1} u_{2} \cdots u_{h}$ the subword decomposition of $w$, and write $m_{p}=m_{p}(w)$ when we want to emphasize the dependence of the sequence $m_{1}, m_{2}, \ldots$ on the word $w$.

It is necessary to partition $Q_{n}(k)$ into more refined hybrid subsets. Let $\alpha$ be a composition of $n$. In other words, $\alpha$ is a vector of positive integers whose components sum to $n$. Say $\alpha=\left(\alpha_{1}, \alpha_{2}, \ldots, \alpha_{h}\right) \in\left(\mathbb{Z}^{+}\right)^{h}$, where

$$
|\alpha|:=\sum_{j=1}^{h} \alpha_{j}=n
$$

For such $\alpha$, let $Q_{n}^{\alpha}(k)$ be a hybrid set consisting of $w \in Q_{n}(k)$, where $m_{p}(w)=2 \sum_{j=1}^{p} \alpha_{j}$ for $1 \leq p \leq h$. Then, by Definition 2 ,

$$
Q_{n}^{\alpha}(k)=\bigcup_{|\tau|=k}^{\cdot} Q_{\alpha_{1}}^{\left(\alpha_{1}\right)}\left(\tau_{1}\right) \times \cdots \times Q_{\alpha_{h}}^{\left(\alpha_{h}\right)}\left(\tau_{h}\right)
$$

where the union is over all compositions $\tau$ of $k$ and the superscript $\left(\alpha_{j}\right)$ in $Q_{\alpha_{j}}^{\left(\alpha_{j}\right)}\left(\tau_{j}\right)$ is the composition of $\alpha_{j}$ with one part. By (14), the left hand side of the equality in (13) becomes

$$
\sum_{w \in Q_{n}(k)} w=\sum_{|\alpha|=n} \sum_{w \in Q_{n}^{\alpha}(k)} w=\sum_{|\alpha|=n} \sum_{|\tau|=k} \prod_{j=1}^{h} \sum_{u_{j} \in Q_{\alpha_{j}}^{\left(\alpha_{j}\right)}\left(\tau_{j}\right)} u_{j}
$$

On the other hand, let $\Delta_{\alpha_{j}}^{\prime}\left(\tau_{j}\right)$ be the subset of $\Delta_{\alpha_{j}}\left(\tau_{j}\right)$ such that $\sum_{l=1}^{m} \pi_{l}=2 m+1$ for any even $m<2 \alpha_{j}$. Then subword decompositions imply that

$$
\sum_{A(w) \in \Delta_{n}(k)} w=\sum_{|\alpha|=n} \sum_{|\tau|=k} \prod_{j=1}^{h} \sum_{A\left(u_{j}\right) \in \Delta_{\alpha_{j}}^{\prime}\left(\tau_{j}\right)} u_{j} .
$$

Hence, it suffices to prove that

$$
\sum_{w \in Q_{n}^{(n)}(k)} w=3^{n} 2^{2 n-k} \sum_{A(w) \in \Delta_{n}^{\prime}(k)} w
$$

Recall that $|w|_{b}=2 n$ and $a(2)+a(4)+\cdots+a(2 n)=k$. We write $w$ as $s_{1} \cdots s_{n}$, where $s_{1}$ contains the letters from the leftmost letter of $w$ up to including $b_{2}$, and $s_{j}$ contains the letters from the letter immediately following $b_{2(j-1)}$ up to including $b_{2 j}$ for $j>1$. Note that any distinct word $w$ in $Q_{n}$ is determined by the sequence $a(i)$ for $1 \leq i \leq 2 n$ from the 
definition of $Q_{n}$. We rewrite the conditions for the sequence $\pi$, where $\pi \in \Delta_{n}^{\prime}(k)$, as

$$
\begin{cases}2 m+1 \leq \sum_{j=1}^{m} \pi_{j} \leq 2 m+2, & \text { if } m \text { is odd, } \\ \sum_{j=1}^{m} \pi_{j}=2 m+1, & \text { if } m \neq 2 n \text { is even, } \\ \sum_{j=1}^{m} \pi_{j}=2 m, & \text { if } m=2 n, \\ \pi_{2}+\pi_{4}+\cdots+\pi_{2 n}=k . & \end{cases}
$$

We will count the number of $w \in Q_{n}^{(n)}(k)$ in (15) with $A(w)=\pi$ for a given sequence $\pi \in \Delta_{n}^{\prime}(k)$.

Let $n=1$. Then $\pi_{1}+\pi_{2}=4$. From (16), $3 \leq \pi_{1} \leq 4$, and $0 \leq \pi_{2} \leq 1$. Thus $w \in$ $\left\{\mid\left(a^{2} b\right) \uplus\left(a^{2} b\right)\right\}$. Now, we count the multiplicity of $w$ in $\left\{\mid\left(a^{2} b\right) \sqcup\left(a^{2} b\right)\right\}$. If $\pi_{1}=4$, then $w=a^{4} b b$. Otherwise, $w=a^{3} b a b$. If $w=a^{4} b b$, then there are $3 \cdot 2^{2} w$ in $\left(a^{2} b\right) \sqcup\left(a^{2} b\right)$. Otherwise, there are only $3 \cdot 2 w$ from the shuffle rule. Hence, there are $3^{n} \cdot 2^{2 n-e(\pi)} w$ in (15), and $w$ comes from $\left\{\mid\left(a^{2} b\right) \sqcup\left(a^{2} b\right)\right\}$.

Let $n \geq 2$. From (16), $\pi_{1}+\pi_{2}=5$ and $\pi_{2 n-1}+\pi_{2 n}=3$. We decompose $w$ into $s_{1} \cdots s_{n}$ and first examine $s_{1}$. There are two cases: $\pi_{1}=4, \pi_{2}=1$ and $\pi_{1}=3, \pi_{2}=2$. In other words, $s_{1}$ is either $a^{4} b a b$ or $a^{3} b a^{2} b$. Thus $s_{1}$ comes from one of the hybrid sets

$$
A_{1}=\left\{a \uplus\left(a^{2} b\right)^{2} \mid\left(a^{2} b\right) a \amalg\left(a^{2} b\right)\right\}
$$

or

$$
A_{2}=\left\{\left(a^{2} b\right)^{2} \sqcup a \mid\left(a^{2} b\right) \sqcup\left(a^{2} b\right) a\right\} .
$$

If $s_{1}$ is $a^{4} b a b$, then it appears on the right of the bars in $A_{1}$ and $A_{2} ; s_{1}$ occurs in $A_{1}$ with multiplicity $6=3 \cdot 2^{2-\pi_{2}}$ by (2). By symmetry, $s_{1}=a^{4} b a b$ occurs in $A_{2}$ with multiplicity 6. On the other hand, if $s_{1}$ is $a^{3} b a^{2} b$, then it appears on both sides in $A_{1}$ and $A_{2}$. Using (2), we easily find that $s_{1}$ occurs with multiplicity 3 on the left sides and multiplicity $3 \cdot 2$ on the right sides in $A_{1}$ and $A_{2}$. Thus the multiplicity of $s_{1}$ becomes $3=3 \cdot 2^{2-\pi_{2}}$ in $A_{1}$ and $A_{2}$ after cancellation.

Similarly, $s_{n}$ is either $a^{3} b b$ or $a^{2} b a b$, which come from one of the hybrid sets

$$
C_{1}=\left\{a b \sqcup a^{2} b\right\} \quad \text { or } \quad C_{2}=\left\{a^{2} b \amalg a b\right\} .
$$

Either of these multi-sets contains $s_{n}=a^{3} b b$ with multiplicity $6=3 \cdot 2^{1-\pi_{2 n}}$ and $s_{n}=a^{2} b a b$ with multiplicity $3=3 \cdot 2^{1-\pi_{2 n}}$.

If $n=2$, then any $w \in Q_{n}^{(n)}$ comes from the hybrid set

$$
A_{1} \times C_{1} \cup A_{2} \times C_{2},
$$


and so its multiplicity in $Q_{n}^{(n)}$ is

$$
3 \cdot 2^{2-\pi_{2}} \cdot 3 \cdot 2^{1-\pi_{4}}+3 \cdot 2^{2-\pi_{2}} \cdot 3 \cdot 2^{1-\pi_{4}}=3^{2} \cdot 2^{4-\pi_{2}-\pi_{4}}=3^{2} \cdot 2^{4-e(\pi)} .
$$

For the cases when $n \geq 3$, we must consider $s_{i}$ with $1<i<n$. From (16), we see that $\pi_{2 i-1}+\pi_{2 i}=4$. Thus $s_{i}$ is either $a^{3} b a b$ or $a^{2} b a^{2} b$, which come from one of the four hybrid sets

$$
\begin{aligned}
& B_{11}=\left\{1 \sqcup\left(a^{2} b\right)^{2} \mid a b a \sqcup a^{2} b\right\}, \\
& B_{12}=\left\{a b\left(a^{2} b\right) \sqcup a \mid a b \sqcup\left(a^{2} b\right) a\right\}, \\
& B_{21}=\left\{a \sqcup a b\left(a^{2} b\right) \mid\left(a^{2} b\right) a \sqcup a b\right\}, \\
& B_{22}=\left\{\left(a^{2} b\right)^{2} \uplus 1 \mid a^{2} b \sqcup a b a\right\} .
\end{aligned}
$$

If $s_{i}=a^{3} b a b$, then it cannot appear on the left sides of the bars in the four hybrid sets above. The multiplicity of $s_{i}=a^{3} b a b$ is $3=3 \cdot 2^{1-\pi_{2 i}}$ in each of these hybrid sets, while that of $a^{2} b a^{2} b$ is 3 in $B_{11}$ and $B_{22}$, and 0 in $B_{12}$ and $B_{21}$ after cancellation, since $s_{i}=a^{2} b a^{2} b$ occurs with multiplicity 1 on the left of the bar and with multiplicity 4 on the right of the bar in $B_{11}$, and it occurs with multiplicity 1 on both sides in $B_{12}$. Then, for example, any $w \in Q_{4}^{(4)}$ comes from the hybrid set

$$
\begin{aligned}
& A_{1} \times B_{11} \times B_{11} \times C_{1} \cup A_{1} \times B_{11} \times B_{12} \times C_{2} \cup A_{1} \times B_{12} \times B_{21} \times C_{1} \cup A_{1} \times B_{12} \\
& \quad \times B_{22} \times C_{2} \cup A_{2} \times B_{21} \times B_{11} \times C_{1} \cup A_{2} \times B_{21} \times B_{12} \times C_{2} \cup A_{2} \times B_{22} \times B_{21} \\
& \quad \times C_{1} \cup A_{2} \times B_{22} \times B_{22} \times C_{2},
\end{aligned}
$$

with each of the $B_{i j}$ occurring in each position exactly one fourth of the time. In general, any $w \in Q_{n}^{(n)}$ comes from the hybrid set

$$
\bigcup_{I} A_{i_{1}}\left(\prod_{j=2}^{n-1} B_{i_{j-1} i_{j}}\right) C_{i_{n-1}}
$$

where $I$ runs over all distinct sequences $\left(i_{1}, \ldots, i_{n-1}\right)$ consisting of 1 's and 2's. Each of the $B_{i j}$ occurs in each position exactly one fourth of the time. We see that the average multiplicity of $s_{i}=a^{3} b a b$ is $3=3 \cdot 2^{1-\pi_{2 i}}$, and of $s_{i}=a^{2} b a^{2} b$ is $3 / 2=3 \cdot 2^{1-\pi_{2 i}}$. Since the decomposition of $Q_{n}^{(n)}$ has $2^{n-1}$ terms, the total multiplicity of $w=s_{1} \cdots s_{n}=$ $a^{\pi_{1}} b a^{\pi_{2}} b \cdots a^{\pi_{2 n-1}} b a^{\pi_{2 n}} b$ in $Q_{n}^{(n)}$ is

$$
2^{n-1} \cdot 3 \cdot 2^{2-\pi_{2}} \prod_{i=2}^{n-1}\left(3 \cdot 2^{1-\pi_{2 i}}\right) \cdot 3 \cdot 2^{1-\pi_{2 n}}=3^{n} \cdot 2^{2 n-e(\pi)}
$$

which completes the proof. 


\section{Acknowledgment}

The author is grateful to David M. Bradley for his help in writing the Introduction, for supplying references, and for several helpful comments. The author thanks Bruce C. Berndt for his advice and encouragement. The author thanks the referee for his helpful comments.

\section{References}

1. J.M. Borwein, D.M. Bradley, and D.J. Broadhurst, "Evaluations of $k$-fold Euler/Zagier sums: A compendium of results for arbitrary k," Elec. J. Comb. 4(2) (1997), \#R5.

2. J.M. Borwein, D.M. Bradley, D.J. Broadhurst, and P. Lisoněk, "Combinatorial aspects of multiple zeta values," Elec. J. Comb. 5(1) (1998), \#R38.

3. J.M. Borwein, D.M. Bradley, D.J. Broadhurst, and P. Lisoněk, "Special values of multiple polylogarithms," Trans. Amer. Math. Soc. 353(3) (2000), 907-941.

4. D. Bowman and D.M. Bradley, "The algebra and combinatorics of shuffles and multiple zeta values," $J$. Combin. Theory Ser. A 97(1) (2002), 43-61.

5. D. Bowman and D.M. Bradley, "Resolution of some open problems concerning multiple zeta evaluations of arbitrary depth," Compositio Mathematica 139(1) (2003), 85-100.

6. D. Bowman and D.M. Bradley, "Multiple polylogarithms: A brief survey," in q-series with Applications to Combinatorics, Number Theory and Physics, B.C. Berndt and K. Ono (Eds.), Contemp Math. American Mathematical Society, Providence, RI, 2001, pp. 71-92.

7. D. Bowman, D.M. Bradley, and J.H. Ryoo, "Some multi-set inclusions associated with shuffle convolutions and multiple zeta values," European J. Combinatorics 24 (2003), 121-127.

8. D.J. Broadhurst and D. Kreimer, "Association of multiple zeta values with positive knots via Feynman diagrams up to 9 loops," Phys. Lett. B 393(3/4) (1997), 403-412.

9. M.E. Hoffman, "Multiple harmonic series," Pacific J. Math. 152(2) (1993), 275-290.

10. M.E. Hoffman, "The algebra of multiple harmonic series," J. Algebra 194 (1997), 477-495.

11. D.E. Loeb, "Sets with a negative number of elements," Adv. Math. 91 (1992), 64-74.

12. M. Waldschmidt, "Valeurs zêta multiples: Une introduction," J. Théorie des Nombres de Bordeaux 12 (2000), 581-595.

13. M. Waldschmidt, "Introduction to polylogarithms," in Number Theory and Discrete Mathematics, A.K. Agarwal, B.C. Berndt, C. Krattenthaler, G.L. Mullen, K. Ramachandra, and W. Waldschmidt (Eds.), Hindustan Book Agency, New Delhi, 2002, pp. 1-12. 